\title{
Diode Laser 805 Hair Removal Side Effects in Groups of Various Ethnicities - Cohort Study Results
}

\author{
Magdalena Atta-Motte ${ }^{*}$, Izabela Załęska² \\ ${ }^{1}$ CQC Compliance Independent Consultant, London, UK \\ ${ }^{2}$ The Section of Professional Cosmetology, The Faculty of Motor Rehabilitation, The University of Physical Education in \\ Krakow, Poland
}

\section{*Correspondence to Magdalena Atta-Motte, M.Sc., MPH, CQC Compliance Independent Consultant, London, UK, 5th Avenue Medical Clinic, 7 Upper Tooting Road, SW17 7TS London, UK. phone +4407932857109 Email: \\ magda.atta.motte@ consultant.com}

Published online March 15, 2020

\begin{abstract}
Introduction: As hair removal has become most popular in aesthetics, the management of its side effects is crucial for every practitioner. Available studies describe the effectiveness of the diode laser hair removal for all skin types according to the Fitzpatrick scale independently, but the question of the occurrence of side effects and adverse effects remains unanswered. This study aims to illustrate aspects of side effects for patients of various ethnicities and the impact of those on the effectiveness of the treatment.

Methods: The research was carried out in Poland and the United Kingdom from March 2016 to March 2019. 217 people of various ethnic origins were qualified for the study, 206 sessions were completed and statistical analysis was performed. The procedure was performed in the pubic area with diode lasers with a wavelength of $805 \mathrm{~nm}$, minimum peak power of $2100 \mathrm{~W}$ and pulse duration between 15 and $400 \mathrm{~ms}$. ET sapphire cooling assisted handle $9 \times 9 \mathrm{~mm}$ large and pulse energy density between 10 and $100 \mathrm{~J} / \mathrm{cm}^{2}$ were used for all treatments. In addition, in order to illustrate the effectiveness of treatments, the subjective and objective analyses of hair loss percentages were indicated. In statistical analysis, the chi-square test for independence was used to evaluate the correlation between the type and severity of side effects and the ethnic origin of patients, the number of treatments, the Fitzpatrick scale and the level of satisfaction. Results: The occurrence of sensitivity depended statistically significantly $(P=0.002)$ on ethnicity. Ethnicity had no significant effect on the occurrence of erythema. The occurrence of hyperpigmentation statistically significantly depended $(P<0.001)$ on ethnicity. The occurrence of burns depended statistically significantly $(P=0.001)$ on ethnicity. The number of treatments had a significant $(P=0.012)$ effect on the severity of side effects occurrence. Among participants who had 6 treatments, only half had side effects, including multiple effects occurring in $9.79 \%$ of cases. The occurrence of side effects such as sensitivity and hyperpigmentation did not depend on the number of treatments. However, the number of treatments had a significant impact on the incidence of erythema $(P<0.001)$ and burns $(P=0.005)$. More than half of the respondents $(58.33 \%)$ had erythema after more than six procedures, whereas in the 6 treatments the erythema occurred only in $6.7 \%$ of cases.

Conclusion: The treatment with the use of diode lasers for all subjects with different ethnicity and thus the recognized scale according to Fitzpatrick is effective and safe. The occurring side effects are transient and do not impact the effect of the whole therapy. Keywords: Side effects; Epilation; Diode laser; Ethnicity; Hair removal.
\end{abstract}

\section{Introduction}

Devices using light, including lasers, have become popular and are commonly used in aesthetics. Aging societies, technological progress, the growing awareness of the use of treatments and increasingly common use of non-invasive procedures, as well as the changing lifestyle and the growing income of patients are factors that drive the market growth of laser therapy in aesthetics.

What is worth noticing in various parts of the world is strict safety regulations for laser use and mandatory qualifications of laser specialists. Such restrictions prevent unauthorized people performing therapy with lasers and IPL, thus minimizing the risks associated with the use of lasers, IPL and LED devices.

Hair reduction, often called hair removal or photoed epilation, is one of the most common laser uses in aesthetics. Lasers used in hair reduction aim to disable effective hair growth by destroying hair bulbs during the anagen growth phase. Lasers such as ruby (694 nm), alexandrite lasers $(755 \mathrm{~nm}$ ) for lighter skin types, Nd: YAG $(1064 \mathrm{~nm})$ for darker skin types and diode lasers (800-810 $\mathrm{nm}$ ) for all skin types I-VI are effective. All these lasers work by using selective photothermolysis, where melanin in the hair follicle is the chromophore.

Hair reduction of $30 \%-50 \%$ was reported in research. ${ }^{1}$ It is well known that despite its widely reported effectiveness, ${ }^{2-8}$ there are certain side effects and unconfirmed adverse effects related to this treatment. ${ }^{9,10}$ Patients who undergo laser hair reduction procedures may expect skin irritation, erythema, edema, postoperative

Please cite this article as follows: Atta-Motte M, Załęska I. Diode Laser 805 hair removal side effects in groups of various ethnicities- cohort study results. / Lasers Med Sci. 2020;11(2):132-137. doi:10.34172/jlms.2020.23. 
hypersensitivity and possible burns manifested by blisters and scabs. It is also possible to experience pigmentary changes such as hyperpigmentation. ${ }^{11,12}$ Less frequently described cases include scarring, purpura, folliculitis, cyanobacteria, pruritus or urticaria. ${ }^{13-15}$

In this article, investigators present the results of a cohort study of side effects occurrence in groups of various ethnicities, as no similar research has been found so far.

\section{The Aim of the Study}

The objective of the study was to investigate the occurrence and types of side effects and adverse events after the use of diode laser $805 \mathrm{~nm}$ for hair removal in groups of various ethnicity patients with I-VI phototypes.

\section{Materials and Methods}

The research was carried out in Poland and the United Kingdom from March 2016 to October 2018.

As shown in Table 1, 247 people took part in the study, of whom 217 - after the initial consultation - were qualified for the course of 6 treatments $(n=217$, females $=174$ ( 1 transgender), $m=43$ ). A course of 6 treatments was completed by 206 participants (females $=168$, males $=39$ ).

One person was excluded from the analysis due to a $100 \%$ success rate of the therapy after 3 treatments. Two females had withdrawn their consent. Therefore, their data is not included in the results. Nine participants discontinued the therapy due to the occurrence of side effects. Twelve participants decided to continue the therapy after they had completed a course of 6 treatments.

Exclusion criteria included: any previous laser or IPL treatments in the study area; cancer; use of hormonal drugs; photosensitizing drugs; antibiotic therapy; use of cosmetics containing retinol, vitamins A, E, C, fruit acids; intake of herbs which can be photosensitizing; suntan; chemical or mechanic depilation or hair bleaching during 6 weeks prior to therapy; irritated skin; dermatosis of various etiology; reticularis; photodermatitis; epilepsy; pregnancy and breastfeeding; isotretinoin use within the past year; history of photosensitivity; history of hypertrophic scars and keloids; age below 20 or above 40 years old.

Table 1. Characteristics of the Group

\begin{tabular}{llcc}
\hline & & Number & Percent \\
\hline \multirow{2}{*}{ Sex } & Female & 172 & 80.0 \\
& Male & 43 & 20.0 \\
Ethnicity & Asian & 29 & 13.5 \\
& Black & 25 & 11.6 \\
\multirow{3}{*}{$\begin{array}{l}\text { Number of } \\
\text { treatments }\end{array}$} & Mixed & 34 & 15.8 \\
& White & 127 & 59.1 \\
& Less than 6 & 9 & 4.2 \\
\hline
\end{tabular}

During initial consultations, according to the study protocol and to ensure patients' safety, investigators assessed skin types using the Fitzpatrick Scale Quiz.

The Fitzpatrick scale is defined as the classification of human skin colour and is used to estimate the response of different types of skin to light exposure. The Fitzpatrick scale is a recognized tool for dermatological research into human skin pigmentation.

Type I always burns, but never tans (pale white; blond or red hair; blue eyes; freckles).

Type II usually burns and tans minimally (white; fair; blond or red hair; blue, green or brown eyes).

Type III sometimes burns mildly and tans uniformly (cream white; fair with any hair or eye color).

Type IV burns minimally and always tans well (moderate brown).

Type $\mathrm{V}$ very rarely burns and tans very easily (dark brown).

Type VI never burns and never tans (intensely pigmented dark brown to darkest brown).

To define ethnicity, in addition to the medical questionnaire, ethnic background questions were asked in accordance with the Census 2001 scheme acknowledged in the United Kingdom.

Several ethnic background types were distinguished in the examined group:

- White: participants with white skin,

- Black: participants with black skin,

- Asian: participants who come from Asia,

- Mixed-race

- White and Black African: participants whose ancestors were white and black and who originally come from Africa.

- White and Black: participants whose ancestors had white and black skin and who come from nonAfrican countries.

- White and Asian: participants whose ancestors had white skin and Asian skin and who come from Asian countries.

A diode laser with a wavelength of $805 \mathrm{~nm}$, the minimum peak power of $2100 \mathrm{~W}$ and pulse duration between 15 and $400 \mathrm{~ms}$, ET sapphire cooling assisted handle $9 \times 9 \mathrm{~mm}$ large, and pulse energy density between 10 and $100 \mathrm{~J} / \mathrm{cm}^{2}$ was used for all treatments.

\section{Patch Test}

This research protocol followed patch tests treatment settings such as fluence $\left(\mathrm{J} / \mathrm{cm}^{2}\right)$ and pulse duration $(\mathrm{ms})$ as per manufacturers' guidelines for different skin types, hair colour, hair and modified according to individual skin reactions. The purpose of a patch test is to evaluate the effects of using the laser at various energy levels on each client's tissue. It takes place on the proposed treatment area and this step cannot be missed according to the treatment protocol.

Patch test settings were starting points for treatments. 
To achieve the best results and to ensure patients' safety (taking into account their ethical history) during each treatment, fluence and pulse width were adjusted to individual participant's skin reaction.

Initial settings and final settings of pulse duration (ms) and fluence $\left(\mathrm{J} / \mathrm{cm}^{2}\right)$ were documented for this study.

During the initial consultation, practitioners had explained a realistic expected outcome of the course of 6 treatments. Participants were familiarized with the definition of permanent hair reduction issued by the FDA and the possible result of the procedure as a long-term, stable reduction in the number of hairs re-growing after the course of treatments.

Participants were aware that hair reduction would last for 4 to 12 months and permanent hair reduction doesn't mean the elimination of all hairs in the treatment area. The reference point for an excellent result was defined to be an $80 \%$ hair loss.

Participants were aware of the possibilities of side effects and adverse effects. Before carrying out the treatment, the patient's skin was shaved and cleaned, and during 6 treatments the patients were not using any other methods of hair removal, as per the therapist's pre- and post-treatment instructions.

Respondents had 6 treatments planned with intervals of 6 weeks - to follow the pubic area hair growth cycle. The duration of the telogen phase in the pubic area is 3 months, and $30 \%$ of hairs are in the anagen phase of growth; therefore, the authors adjusted the study protocol to the manufacturer's guidance.

Subjective and objective percentage assessments of hair reduction were indicated.

In the objective method, reliable evaluation of hair reduction was introduced by taking photographs (magnified $\times 20$ ) of a $1 \mathrm{~cm}^{2}$ area, placed $4 \mathrm{~cm}$ below the point located right in the middle between iliac spines. Hairs were counted and the percentage of hair reduction was assessed before the first treatment and 6 weeks after the last one was carried out. The number of hairs in each photo was counted by using magnified screen images.

Three evaluators independently counted the hairs and the mean value was used for the evaluation. To maintain evaluation neutrality, no information was given to evaluators about the participants or the chronology of the photographs. The hair reduction measure in this study was defined as the number of removed hairs divided by the initial number of pubic hairs, expressed as a percentage.

Simultaneously, 6 weeks after completing the treatment cycle, the patients were asked to share their opinion on hair loss percentage and to estimate the percentage of hair loss in the treated area. The results of this part of the cohort research were published. ${ }^{16}$

Before and after each treatment, in cooperation with the participants, the side effects were defined as follows:

- skin hyperpigmentation was defined as the excessive pigmentation of the skin which was reported by participants and confirmed by investigators through picture comparison.

- skin redness, also known as Erythema (from the Greek erythros, meaning red), is the redness of the skin caused by hyperemia (increased blood flow) in superficial capillaries. It occurs with any skin injury, infection, or inflammation. It was reported by participants when lasting longer than 48 hours after the treatment.

- $\quad$ skin irritation was defined as itchiness combined with edema and erythema. It was reported by participants when lasting longer than 48 hours after the treatment.

- skin sensitivity was defined as the indication of slight changes in skin condition and it was subjectively reported by participants.

- skin burns were defined as skin injury due to overheating, reported by participants when occurred and treated according to the adverse effect policy and treatment protocol.

Adverse effects were defined as: discomfort, damage to the natural skin texture, scarring, excessive swelling, blisters, bruising. They were documented when observed.

Treatment settings, such as fluence and pulse duration, were documented.

The analysis of a part of this cohort study, focused on a group of mixed-race patients, has been published. ${ }^{17}$

\section{Statistics}

The chi-square test for independence was used to evaluate the correlation between the type and severity of side effects and the ethnic origin of patients, the number of treatments, the Fitzpatrick scale and the level of satisfaction.

After 6 treatments, the mean values of hair loss percentage from the subjective evaluation by examined patients were compared with the mean values of hair loss percentage in the objective assessment of the participants in the groups with different intensity of adverse effects using ANOVA.

Similarly, after 6 treatments, the mean values of hair loss percentage from the subjective evaluation by examined patients were also compared with the mean values of hair loss percentage in the objective assessment of the participants in the groups where selected types of adverse effects did or did not occur, as measured by the Student's t-test for independent groups.

Statistically significant results were assumed to be those of significance level equal to or lower than 0.05 ( $P$ $\leq 0,05)$. The lack of statistical significance was marked with the abbreviation NS (i.e. Statistically Insignificant). Calculations were made using statistical software STATISTICA 13.

\section{Results}

Figure 1 shows that $53 \%$ of the participants had side effects, $40.9 \%$ of the participants had single side effects 
Increased occurrence of the side effects

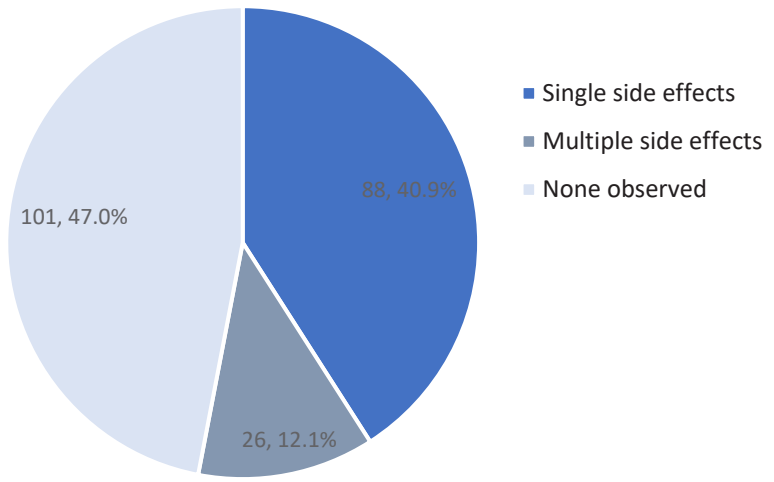

Figure 1. Increased Occurrence of the Side Effects.

and $12.10 \%$ had multiple side effects.

A detailed breakdown of side effects was observed and documented in this study: $44.1 \%$ sensitivity, $24.1 \%$ burns, $24.1 \%$ hyperpigmentation, and $14.5 \%$ erythema. As irritation was observed in $2.8 \%$ of the participants with side effects, it was not included in the further analysis as shown in Figure 2.

The severity of side effects occurrence depended statistically significantly $(P<0.001)$ on the ethnicity of the participants. The side effects were observed more frequently in the groups of Black and Mixed-race participants. Among the Black and Mixed-race groups of participants, multiple side effects were ten times more frequent than in the White and Asian groups of participants as shown in Figure 3.

The correlation of ethnicity with types of side effects occurrence, as it is shown in Table 2, is as follows:

- The occurrence of sensitivity depended statistically significantly $(P=0.002)$ on ethnicity.

- Ethnicity had no significant effect on the occurrence of erythema.

- The occurrence of hyperpigmentation statistically significantly depended $(P<0.001)$ on ethnicity.

- The occurrence of burns depended statistically significantly $(\mathrm{P}=0.001)$ on ethnicity.

The correlation of severity of side effects occurrence with the number of treatments is shown in Figure 4 as follows:

The number of treatments had a significant $(P=0.012)$ effect on the severity of side effects occurrence. Among participants who had 6 treatments, only half had side effects, including multiple effects occurring in $9.79 \%$ of cases.

Most of the side effects occurred in the group of participants who had more than 6 treatments. In this group, single side effects occurred in half of the subjects and multiple side effects in one-third of the participants.

The correlation of the number of treatments with the types of side effects is shown in Table 3 as follows:

\section{The frequency of side effects occurrence}

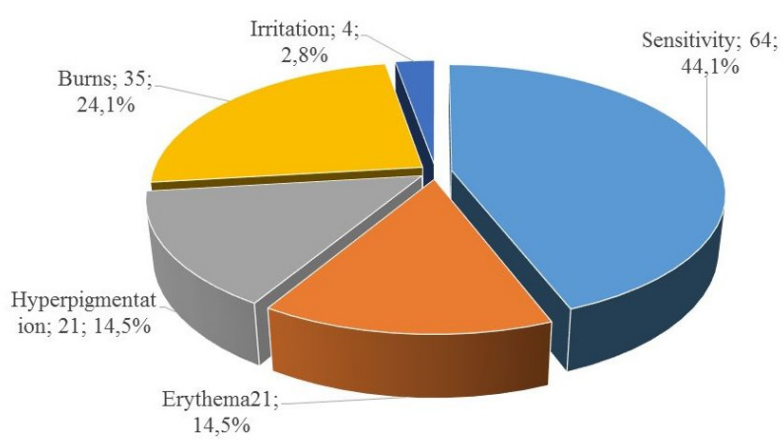

Figure 2. The Frequency of Side Effects Occurrence. * Due to the small number of cases, Irritation was not included in further analysis.

The severity of the occurrence of side effects and ethnicity

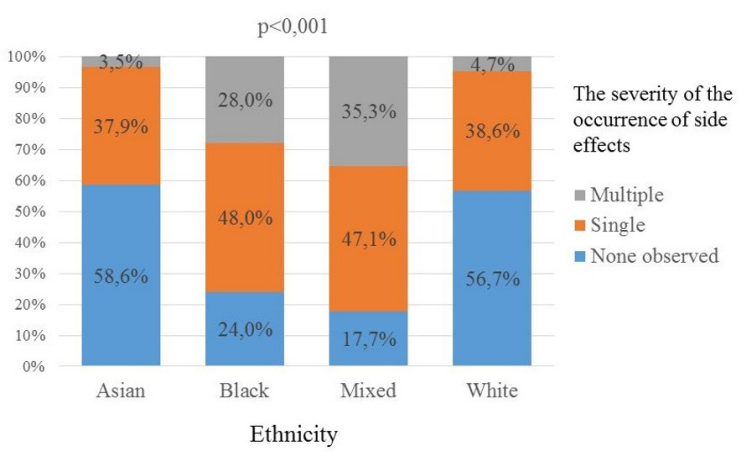

Figure 3. The Frequency of Side Effects Occurrence.

Table 2. The Occurrence of Side Effects and Ethnicity

\begin{tabular}{|c|c|c|c|c|}
\hline \multirow[t]{2}{*}{ Ethnicity } & \multicolumn{4}{|c|}{ Types of Side Effects } \\
\hline & Sensitivity & Erythema & Hyperpigmentation & Burns \\
\hline \multirow{2}{*}{$\begin{array}{l}\text { Asian } \\
(n=29)\end{array}$} & 5 & 2 & 3 & 3 \\
\hline & $17.24 \%$ & $6.90 \%$ & $10.34 \%$ & $10.34 \%$ \\
\hline \multirow{2}{*}{$\begin{array}{l}\text { Black } \\
(n=25)\end{array}$} & 12 & 0 & 7 & 9 \\
\hline & $48.00 \%$ & $0.00 \%$ & $28.00 \%$ & $36.00 \%$ \\
\hline \multirow{2}{*}{$\begin{array}{l}\text { Mixed } \\
(n=34)\end{array}$} & 17 & 4 & 11 & 10 \\
\hline & $50.00 \%$ & $11.76 \%$ & $32.35 \%$ & $29.41 \%$ \\
\hline \multirow{2}{*}{$\begin{array}{l}\text { White } \\
(n=127)\end{array}$} & 30 & 15 & 0 & 13 \\
\hline & $23.62 \%$ & $11.81 \%$ & $0.00 \%$ & $10.24 \%$ \\
\hline $\begin{array}{l}\text { Significance of } \\
\text { differences }\end{array}$ & $P=0.002$ & NS & $P<0.001$ & $P=0.001$ \\
\hline
\end{tabular}

The occurrence of side effects such as sensitivity and hyperpigmentation did not depend on the number of treatments.

The number of treatments had a significant impact on the incidence of erythema $(P<0.001)$ and burns $(P=0.005)$. More than half of the respondents $(58.33 \%)$ had erythema after more than 6 procedures, whereas in 


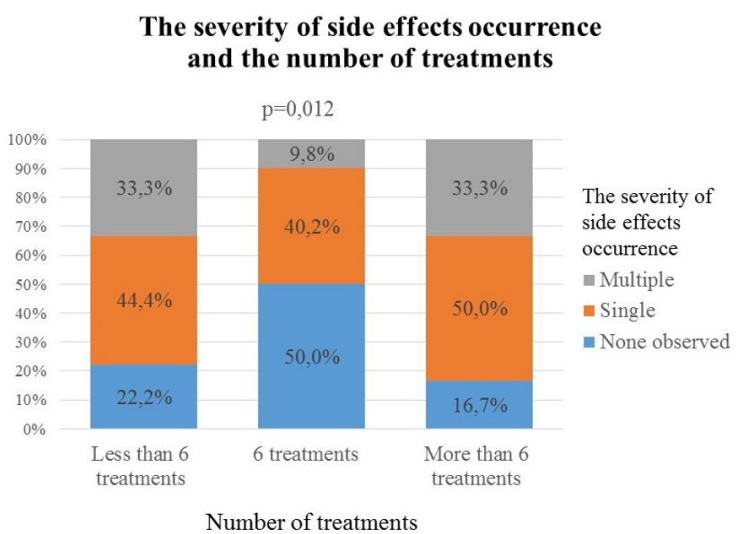

Figure 4. The Severity of Side Effects Occurrence and the Number of Treatments.

Table 3. The Occurrence of Side Effects and Ethnicity

\begin{tabular}{lcccc}
\hline $\begin{array}{l}\text { No. } \\
\text { of Treatments }\end{array}$ & \multicolumn{4}{c}{ Types of Side Effects } \\
\cline { 2 - 5 } & Sensitivity & Erythema & Hyperpigmentation & Burns \\
\hline $\begin{array}{l}<6 \\
(\mathrm{n}=9)\end{array}$ & 3 & 1 & 1 & 5 \\
\hline 6 & $33.33 \%$ & $11.11 \%$ & $11.11 \%$ & $55.56 \%$ \\
$(\mathrm{n}=194)$ & 58 & 13 & 19 & 28 \\
\hline 6 & $29.90 \%$ & $6.70 \%$ & $9.79 \%$ & $14.43 \%$ \\
$(\mathrm{n}=12)$ & 3 & 7 & 1 & 2 \\
\hline $\begin{array}{l}\text { Significance of } \\
\text { differences }\end{array}$ & $25.00 \%$ & $58.33 \%$ & $8.33 \%$ & $16.67 \%$ \\
\hline
\end{tabular}

the 6 treatments, the erythema occurred only in $6.7 \%$ of cases.

More than half of the respondents (55.56\%) who discontinued the treatments (less than 6 treatments) had burns, whereas burns occurred only in $14.43 \%$ of cases in the group of the participants who had 6 treatments.

\section{Discussion}

The review of available existing references, including Fayne et al in $2018^{18}$ indicates that diode lasers, alexandrite lasers, Nd:YAG, ruby lasers and some sources of IPL are safe for hair reduction with minimal side effects in groups of IV-VI skin type patients as long as proper treatment protocols and energy settings are used.

This study shows that side effects in diode laser 805 $\mathrm{nm}$ hair reduction are common in groups of various ethnicities among skin types I-VI where treatment protocols by manufacturers' guidelines were followed and proper pre-caution was applied.

This study shows that single or multiple side effects depend statistically significantly on the ethnicity of participants. Multiple side effects were observed ten times more frequently in the groups of Black and Mixedrace participants than in the White and Asian groups of participants. Investigators have noted that the LES scale should be used in addition to the Fitzpatrick scale to ensure patients' safety. ${ }^{19}$

For a long time, it has been noted that temporary erythema and post-inflammatory hyperpigmentation are the most frequent short-time side effects as shown by Boss et al in $1999,{ }^{20}$ Lim \& Lanigan in $2006^{9}$ and Gan \& Graber in $2013^{11}$ in their studies.

This study shows side effects such as sensitivity, erythema, hyperpigmentation and burns depend on patients' ethnicity. It is well known that laser hair reduction causes no long-term side effects nor adverse effects as reported by Lim and Lanigan in $2006^{9}$ or Nistico et al in $2018 .^{21}$

Investigators found no adverse effects in this study. Most of the reviews have focused on lighter skin types and excluded darker skin types as those types are much more vulnerable and face a high risk of unwanted side effects such as pigmentation changes, blisters, and crust formation as shown by Fayne et al in 2018. ${ }^{17}$

This study reports no blisters or crust formation in darker skin patients. In the group of black skin patients, $28 \%$ noted hyperpigmentation. This result is different in the study by Vachiramon et al in 2012, ${ }^{22}$ where hyperpigmentation was noted among $6 \%$ of the patients. They have also noted blistering or scarring which was not observed in this study. Psoriasis was reported by Garg et al as a rare occurrence in $2018 .{ }^{23}$ In this article the authors underlined the importance of patient ethnicity.

\section{Conclusion}

This study shows a correlation between ethnicity and side effects occurrence after diode laser hair removal treatments in the pubic area. The groups of Black and Mixed-race participants had much more frequent multiple side effects compared to the groups of White and Asian participants.

Sensitivity, erythema and burns as side effects were observed. No adverse effects were seen during this study. Among all observed side effects, only erythema had no statistical correlation with ethnicity; however, it was not observed in the group of Black participants. Hyperpigmentation was not found among the White participants.

\section{Ethical Considerations}

All procedures involving human participants in this study were performed following the ethical standards of the institutional and/or national research committee and with the 1964 Helsinki declaration and its later amendments or comparable ethical standards. The study has been registered in the ISRCTN registry as a cohort study (identifier: ISRCTN10288390).

\section{Conflict of Interests}

The authors declare no conflict of interest. 


\section{References}

1. Liew SH. Laser Hair Removal: guidelines for management. Am J Clin Dermatol. 2002;3(2):107-15. doi: 10.2165/00128071-200203020-00004.

2. Pavlović MD, Adamič M, Nenadić D. Fixed, low radiant exposure vs. incremental radiant exposure approach for diode laser hair reduction: a randomized, split axilla, comparative single-blinded trial. J Eur Acad Dermatol Venereol. 2015;29(12):2377-81. doi: 10.1111/jdv.13239.

3. Tremaine AM, Avram MM. FDA MAUDE data on complications with lasers, light sources, and energy-based devices. Lasers Surg Med. 2015;47(2):133-40. doi:10.1002/ lsm.22328.

4. Ormiga $\mathrm{P}$, Ishida $\mathrm{CE}$, Boechat $\mathrm{A}$, Ramos-E-Silva $\mathrm{M}$. Comparison of the effect of diode laser versus intense pulsed light in axillary hair removal. Dermatol Surg. 2014;40(10):1061-9. doi: 10.1097/DSS.0000000000000138.

5. Koo B, Ball K, Tremaine AM, Zachary CB. A comparison of two 810 diode lasers for hair removal: Low fluence, multiple pass versus a high fluence, single pass technique. Lasers Surg Med. 2014;46(4):270-4. doi: 10.1002/lsm.22226.

6. AL-Hamamy HR, Saleh AZ, Rashed ZA. Evaluation of effectiveness of diode laser system (808 nm) versus Intense Pulse Light (IPL) in the management of unwanted hair: A split face comparative study. Int J Med Phys Clin Eng Radiat Oncol. 2015;4(1):41-8. doi: 10.4236/ijmpcero.2015.41006.

7. Krauss MC. Removal of bikini hair using a rapid 810-nm diode laser. Cosmet Dermatol. 2008;21(7):392-396.

8. Jo SJ, Kim JY, Ban J, Lee Y, Kwon O, Koh W. Efficacy and safety of hair removal with a long-pulsed diode laser depending on the spot size: A randomized, evaluatorsblinded, left-right study. Ann Dermatol. 2015;27(5):517-22. doi: 10.5021/ad.2015.27.5.517.

9. Lim SP, Lanigan SW. A review of the adverse effects of laser hair removal. Lasers Med Sci. 2006; 21(3):121-5. doi: 10.1007/s10103-006-0377-y.

10. Tejero P, Sunkel V, Zamorano MV. Adverse effects of laser hair removal. J Surg. 2015;3(1-1):18-20. doi: 10.11648/j. js.s.2015030101.16.

11. Gan SD, Graber EM. Laser hair removal: a review. Dermatol Surg. 2013;39(6):823-38. doi: 10.1111/dsu.12116.

12. Ibrahimi OA, Avram MM, Hanke CW, Kilmer SL, Anderson RR. Laser hair removal. Dermatol Ther. 2011;24(1):94-107. doi: 10.1111/j.1529-8019.2010.01382.x.
13. Royo J, Urdiales F, Moreno J, Al-Zarouni M, Cornejo P, Trelles MA. Six-month follow-up multicenter prospective study of 368 patients, phototypes III to V, on epilation efficacy using an 810-nm diode laser at low fluence. Lasers Med Sci. 2011;26(2):247-55. doi:10.1007/s10103-010-08461.

14. Agarwal M, Velaskar S, Gold MH. Efficacy of a low fluence, high repetition rate $810 \mathrm{~nm}$ diode laser for permanent hair reduction in indian patients with skin types IV-VI. J Clin Aesthet Dermatol. 2016;9(11):29-33.

15. Wanner M. Laser hair removal. Dermatol Ther. 2005;18(3):209-16. doi:10.1111/j.1529-8019.2005.05020.x

16. Atta-Motte M, Załęska, I. Effectiveness of diode laser 805 $\mathrm{nm}$ in pubic hair removal in groups of various ethnicity. Pol J Cosmetol. 2018; 21(2): 177-183.

17. Załęska I, Atta-Motte M. Aspects of diode laser (805 nm) hair removal safety in a mixed-race group of patients. J Lasers Med Sci. 2019;10(2):146-52. doi:10.15171/ jlms.2019.23.

18. Fayne RA, Perper M, Eber AE, Aldahan AS, Nouri K. Laser and light treatments for hair reduction in Fitzpatrick skin types IV-VI: A comprehensive review of the literature. Am J Clin Dermatol. 2018;19(2):237-52. doi: 10.1007/s40257017-0316-7.

19. Lancer HA. Lancer ethnicity scale (LES). Lasers Surg Med. 1998;22:9. doi:10.1002/(SICI)10969101(1998)22:1<9::AID-LSM4>3.0.CO;2-T.

20. Boss WK Jr, Usal H, Thompson RC, Fiorillo MA. A comparison of the long-pulse and short-pulse Alexandrite laser hair removal systems. Ann Plast Surg. 1999; 42(4): 381-4. doi: 10.1097/00000637-199904000-00006.

21. Nistico SP, Del Duca E, Farnetani F, Guida S, Pellacani G, Rajabi-Estarabadi A, et al. Removal of unwanted hair: efficacy, tolerability, and safety of long-pulsed 755-nm alexandrite laser equipped with a sapphire handpiece. Lasers Med Sci. 2018;33(7):1479-83. doi: 10.1007/s10103018-2503-Z.

22. Vachiramon V, Brown T, McMichael AJ. Patient satisfaction and complications following laser hair removal in ethnic skin. J Drugs Dermatol. 2012;11(2):191-5.

23. Garg S, Borde Bisht P, Rao S. Psoriasis postlaser hair removal. Dermatol Surg. 2018;44(4):602-3. doi: 10.1097/ DSS.0000000000001263. 